\title{
Impact of COVID-19 On Breast Cancer Management: A Radiological Prespective from A Tertiary Centre
}

(D) Anjum Syed, (1) Gangotri Kumari, (10) Aakriti Kapoor, (1) Satish Chaitanya, (1) Prateek Sharda, (D) Mriganki Chaudhary,

(1) Ananya Deori, (1) Priyanka Gupta, (1) Nilotpal Choudhary, (1) Shalinee Rao, (1) Bina Ravi

Department of Radiodiagnosis, All India Institute of Medical Science Rishikesh, Rishikesh, India

\begin{abstract}
Objective: The coronavirus-2019 (COVID-19) pandemic caused delaying breast cancer management, increasing time interval in chemotherapy cycles and surgery. This has implications on radiological manifestation of cancer. Further, we evaluated changes observed in mammography.

Materials and Methods: This case control study was conducted from March 25 th 2020 to August 15 th 2020 at the Integrated Breast Care Centre, All India Institute of Medical Science Rishikesh (AIIMS), Rishikesh. Sonomammography was performed on follow-up patients who were on chemotherapy and were scheduled for surgery. Moreover, duration of delay from the last neoadjuvant chemotherapy (NACT) cycle was recorded. Similar data in the pre-COVID-19 period from November $4^{\text {th }}, 2019$ to March 24 $4^{\text {th }}, 2020$ was compared with post-COVID-19 data and was analyzed by SPSS Version 23.

Results: The study included 54 patients who presented between March 25 th 2020 and August $15^{\text {th }}, 2020$. Furthermore, the delay in NACT cycles has been shown to be associated with disease progression $(p=0.045)$. Subgroup analysis of treatment duration with various parameters revealed significant correlation between size, appearance of ulceration, and response evaluation $(\mathrm{p}<0.05)$. However, no significant association was found between duration of delay and the histological subtype of lesion ( $p>0.05)$. A substantial difference was seen in the evaluation of NACT response in pre- and post-COVID-19 time, with partial response $(n=39,58.24 \%)$ seen as the most common response in pre-COVID-19 time and progressive disease $(n=28,51.9 \%)$ as the most common response in post-COVID-19 time $(\mathrm{p}<0.001)$.
\end{abstract}

Conclusion: The coronavirus pandemic has severe impact on breast cancer management. A delay in NACT causes progression in cancer. This can be seen in ultrasound and mammogram.

Keywords: Breast cancer, neoadjuvant chemotherapy, delay, mammography

Cite this article as: Syed A, Kumari G, Kapoor A, Chaitanya S, Sharda P, Chaudhary M, Deori A, Gupta P, Choudhary N, Rao S, Ravil B. Impact of COVID-19 On Breast Cancer Management: A Radiological Prespective from A Tertiary Centre. Eur J Breast Health 2021; 17(2): 180-187

\section{Key Points}

- Coronavirus pandemic has severe impacts on breast cancer management.

- The delay in neoadjuvant chemotherapy cycles and duration in between the neoadjuvant chemotherapy cycles and surgery is associated with disease progression.

- The impacts of delay in neoadjuvant chemotherapy cycles can have radiological manifestations which can be seen on mammography and ultrasound.

\section{Introduction}

In the year 2020, the coronavirus pandemic affected many countries worldwide. One of the hardest hit countries is India. To cope up with such a pandemic, nationwide lockdown was imposed from March 25 ${ }^{\text {th }}, 2020$ to May 31 $1^{\text {st }}, 2020$ for a period of 68 days (1). This restricted the movement of people, which was necessary given the pandemic situation. It was also recommended that cancer surgery be postponed and chemotherapy be continued in cancer patients to reduce the risk of hospital-acquired coronavirus disease-2019 (COVID-19). Moreover, health emergencies and cancer treatment were exempted from the stringent lockdown. However, lack of awareness caused delays in the management of various cancer patients, such as those who were on chemotherapy and radiotherapy and were scheduled for surgery. Furthermore, the effects of delays in treatment due to such a pandemic situation can be seen on imaging in these cancer patients.

Many studies have been carried out worldwide, such as those by Freer (2) and Broom et al. (3), which state that the coronavirus pandemic has adversely affected the cancer management in general and breast cancer in particular. As a precautionary measure to reduce the infection, the level 


\section{Syed et al. Impact of COVID-19 on Breast Cancer Management}

of care provided to cancer patients has reduced since the pandemic (4). The management of patients with breast cancer has been impaired, resulting in delayed diagnosis, chemotherapy, and surgery. Timelines from surgery to adjuvant chemotherapy has been increased $(5,6)$. In addition, breast cancer imaging was affected, showing a marked reduction in case volumes, especially during the first 2 months of the pandemic. All imaging modalities, such as mammography, ultrasonography, and breast magnetic resonance imaging (MRI), were affected; however, breast mammography was most affected $(2,7)$.

About half a century ago, preoperative chemotherapy was started with the goal of treating locally advanced and non-operable breast cancer (8). However, preoperative NACT is also regarded as the standard of care for primary operable breast cancer these days (9). The objectives of preoperative NACT are not only to minimize cancer and increase breast-conserving surgery but also to achieve pathological complete response $(8,10)$.

Various imaging modalities are available for assessing the response evaluation of NACT in patients with breast cancer, such as physical examination in conjunction with mammography and ultrasonography, dynamic breast MRI, Positron emission tomography scan, and mammoscintigraphy $(11,12)$. Out of the various available modalities, physical examination, together with mammography and ultrasonography, is most widely used modality (12).

The latest guideline for response evaluation in solid tumors is response evaluation criteria in solid tumors (RECIST) Version 1.1 (13). In compliance with this guideline, the response evaluation to treatment can be provided in the form of complete response, partial response, progressive disease, and stable disease after comparison with prior imaging.

The main objective of this case control study is to evaluate changes observed in mammography and ultrasonography due to delays in the treatment course of breast cancer. This entails the delay in neoadjuvant chemotherapy cycles and duration gap between the last neoadjuvant chemotherapy and surgery.

\section{Materials and Methods}

This case control study was conducted at the Integrated Breast Care Centre, AIIMS, Rishikesh, from March $25^{\text {th }}$, 2020 to August $15^{\text {th }}$, 2020 for a period of 4.5 months. This data was correlated with the data collected from November $04^{\text {th }}, 2019$ to March $24^{\text {th }}, 2020$ taken in pre-COVID-19 time. Further, the response evaluation was performed according to RECIST Version 1.1. The duration of delay from the last NACT cycle was recorded. There were also histopathological types of each patient obtained. In addition, changes observed in mammogram and ultrasound were recorded. This included changes in size, change in density, and appearance of new calcification, satellite nodules, and lymph node status and muscle/skin infiltration. Mammography was performed using the Hologic Selenia Dimensions, Hologic (USA). On the other hand, ultrasound was performed using 4-12 $\mathrm{MHz}$ linear high-frequency probe of Esaote MyLab 9 eXP Diagnostic Ultrasound system, Model MyLab 9 eXP scanner.

\section{Inclusion criteria:}

- All follow-up patients who were on NACT and visited again for response evaluation.

- All follow-up patients who have completed their NACT and were scheduled for surgery.

\section{Exclusion criteria:}

- All new patients who came for the first time.

- All benign cases.

- All those patients who had received their prior neoadjuvant therapy and presented for future follow-up at our center for the first time were excluded because there was no prior imaging available for comparison.

The analysis of data was conducted using SPSS Version 23. For descriptive statistics, the data was analyzed, and the association between various parameters was correlated using non-parametric tests of correlation, such Spearman's rank-order correlation coefficient, Kruskal-Wallis $\mathrm{H}$ test, and Mann-Whitney-Wilcoxon test as the duration of delay was not normally distributed.

\section{Results}

\section{Patient characteristics}

A total of 54 patients presented for follow-up between March 25 ${ }^{\text {th }}, 2020$ and August $15^{\text {th }}, 2020$. This post-COVID-19 data was contrasted with pre-COVID-19 data collected from November $4^{\text {th }}, 2019$ to March $24^{\text {th }}, 2020$. The duration of delay, associated RECIST, histological subtypes of tumor, and radiological findings during both periods are summarized in Table 1. It was found that in the pre-COVID-19 times, the patient was found to have a mean duration delay of 15.69 days ( median $=14$ days and mode $=10$ days) from the last NACT cycle and, if scheduled for surgery, was usually operated within 6 weeks ( 42 days). However, during the post-COVID-19 times, this duration has increased, and patients used to present a mean duration delay of 85.76 days $($ median $=89$ days and mode $=72$ days), increasing the time duration between the NACT cycles and gap between the last NACT cycle and surgery. Due to such a long gap of duration, most patients (n $=28,51.9 \%$ ) developed progressive diseases and had to undergo a few more NACT cycles before undergoing surgery. The maximum duration of delay observed during the pre-COVID-19 time was 50 days post 4 cycles of NACT, while the minimum duration of delay was 2 days post 4 cycles of NACT. However, during the post-COVID-19 times, the maximum duration of delay was 117 days observed in patients post 9 cycles of NACT, whereas the minimum duration of delay was 29 days post 10 cycles of NACT. During the post-COVID-19 time, the average duration patients underwent surgery following their last NACT cycle was 73 days if the post-NACT mammogram and ultrasonography suggested a stable disease or partial response.

When considering the post-COVID-19 period, the average age of presentation was 45.35 years, with standard deviation of 10.60 years. Most patients had triple-negative breast cancer $(n=15,27.8 \%)$, with malignant phyllodes $(n=2,3.7 \%)$ being the least common tumor seen. Moreover, progressive disease was mostly seen in triple-negative breast cancer $(\mathrm{n}=11,20)$. Right- and left-sided breast involvement was seen in 27 cases $(50 \%)$ each.

In comparison with pre-COVID-19 time, the most common response seen was partial response $(\mathrm{n}=39,58.2 \%)$, while the least common was complete response $(\mathrm{n}=1,1.5 \%)$. Further, progressive disease was seen in only seven cases $(10.4 \%)$.

Increasing the duration between the NACT cycles and the last NACT cycle and surgery is strongly correlated with disease progression (Table 1 ). In the response evaluation, a significant statistical difference was observed in pre- and post-COVID-19 time $(\mathrm{p}<0.001)$. 
Table 1. Summary of all parameters

Pre-COVID-19

All parameters

Age (years)

Duration (days)

Laterality

Right

Left

Bilateral

Cycles of NACT

No NACT

2 cycles

4 cycles

5 cycles

6 cycles

7 cycles

8 cycles

9 cycles

10 cycles

11 cycles

12 cycles

Change in size

Increased

Decreased

Stable

Change in density

Increased

Decreased

Stable

Mammogram not done

Calcification in lesion

New calcification

Same as prior

No calcification

Mammogram not done

\section{Appearance of ulceration}

New ulceration

Ulcerated since earlier

No ulceration

Ipsilateral axillary lymph nodes

New nodes

Same as previous

No nodes

Reduced
Mean \pm SD ||min-max\| Frequency

(\%)

$44.54 \pm 9.01|| 24-63 \|$

$15.69 \pm 8.88|| 2-50 \|$

39 (58.2\%)

$27(40.3 \%)$

$1(1.5 \%)$

$$
\begin{aligned}
& 0 \\
& 0
\end{aligned}
$$

$33(49.3 \%)$

$4(6.0 \%)$

1 (1.5\%)

0

27 (40.3\%)

0

0

0

2 (30\%)

9 (13.4\%)

57 (85.1\%)

1 (1.5\%)

1 (1.5\%)

25 (37.3\%)

40 (59.7\%)

1 (1.5\%)

0

28 (41.8\%)

38 (56.7\%)

1 (1.5\%)

0

1 (1.5\%)

66 (98.5\%)

0

30 (44.8\%)

28 (41.8\%)

$9(13.4 \%)$
Post-COVID-19

Mean \pm SD ||min-max\| Frequency

(\%)

$45.35 \pm 10.60$ ||28-66||

$85.76 \pm 33.29|| 29-136 \|$

27 (50\%)

27 (50\%)

0

$3(5.6 \%)$

$1(1.9 \%)$

$13(24.1 \%)$

$2(3.7 \%)$

0

$3(5.6 \%)$

27 (50\%)

1 (1.9\%)

$3(5.6 \%)$

$1(1.9 \%)$

0

$26(48.1 \%)$

$19(35.2 \%)$

9 (16.7\%)

15 (27.8\%)

7 (13\%)

24 (44.4\%)

8 (14.8\%)

5 (9.3\%)

16 (29.6\%)

25 (46.3\%)

8 (14.8\%)

4 (7.4\%)

3 (5.6\%)

47 (87\%)

8 (14.8\%)

28 (51.9\%)

18 (33.3\%)

0 
Table 1. Continued

Pre-COVID-19

Mean \pm SD \|min-max\| Frequency

(\%)

All parameters
Infraclavicular lymph nodes
New nodes
Same as previous
No nodes
Supraclavicular lymph nodes
New nodes
Same as prior
No nodes
Contralateral side
New infiltrated axillary LN
No change
New malignant breast lesion
Histological subtype
Luminal type A
ER/PR/HER2neu+ve
HER 2neu+ve
TNBC

Phyllodes tumor

RECIST

Stable

$$
\begin{gathered}
20(29.9 \%) \\
7(10.4 \%) \\
39(58.2 \%) \\
1(1.5 \%)
\end{gathered}
$$

Progressive disease

Partial response

Complete response

\section{Post-COVID-19}

Mean \pm SD ||min-max\| Frequency

(\%)

LN: Lymph nodes; SD: Standard deviation; ER: Estrogen receptor; PR: Progesterone receptor; HER2: Human epidermal growth factor receptor 2; TNBC: Triple-negative breast cancer; COVID-19: Coronavirus disease-2019; min: Minimum; max: Maximum; RECIST: Response evaluation criteria in solid tumors

Impact of duration of delay in treatment on various parameters during post-COVID-19 times

Most patients were able to visit the hospital after a median duration delay of 89 days ( mode $=72$ days, mean $=85.76$ days). We analyzed the impact of duration of delay (days) in treatment on the characteristics of various patients, such as age of presentation, side of breast involvement, status of the NACT cycles, and appearance of ulceration. In addition, we have also studied its impact on radiological features, such as change in size, mammographic density, presence of calcification, and lymph nodal status. A correlation with histological subtype and RECIST was also carried out. The following were our observations:

- A significant correlation $(\mathrm{p}<0.05)$ was observed in relation to the breast involved, change in size of lesion, appearance of ulceration, infraclavicular lymph nodes, and RECIST (Table 2).

- Change in size of lesion: It was found that radiological increase in size of lesion was seen in $48.1 \%$ of cases $(n=26, p=0.042)$, whereas a decrease was seen in $35.2 \%$ of cases $(n=19)$.
- Appearance of new ulceration: Delay in treatment was seen to be associated with the appearance of new ulceration in lesion in $7.4 \%$ of cases $(n=4, p=0.013)$. As a result of ulceration, mammography could not be performed in these patients.

- RECIST: On response evaluation, the majority of patients displayed progressive disease (Figure 1$)(\mathrm{n}=28,51.9 \%, \mathrm{p}=0.045)$, whereas the least commonly seen was complete response $(\mathrm{n}=1,1.9 \%)$.

- Other characteristics: Other radiological changes were also seen, such as the appearance of a new calcification $(n=5,9.3 \%, p=0.223)$ and increase in mammographic density of lesion $(\mathrm{n}=15,027.8 \%)$ (Figure 2); however, this correlation was insignificant ( $\mathrm{p}=0.802$ ) (Table 2). The appearance of new malignant lesion in contralateral breast $(\mathrm{n}=5,9.3 \%)$ (Figure 3) and new lymph nodes in contralateral axilla $(\mathrm{n}=2,3.7 \%)$ was found in few cases $(\mathrm{p}=0.166)$.

\section{Discussion and Conclusion}

Breast cancer is the most common malignancy in females, with a lifetime risk of up to 1.8 (14). Before surgery, neoadjuvant therapies, 
Table 2. Non-parametric correlation analysis between delay in duration (days) and post-COVID-19 parameters

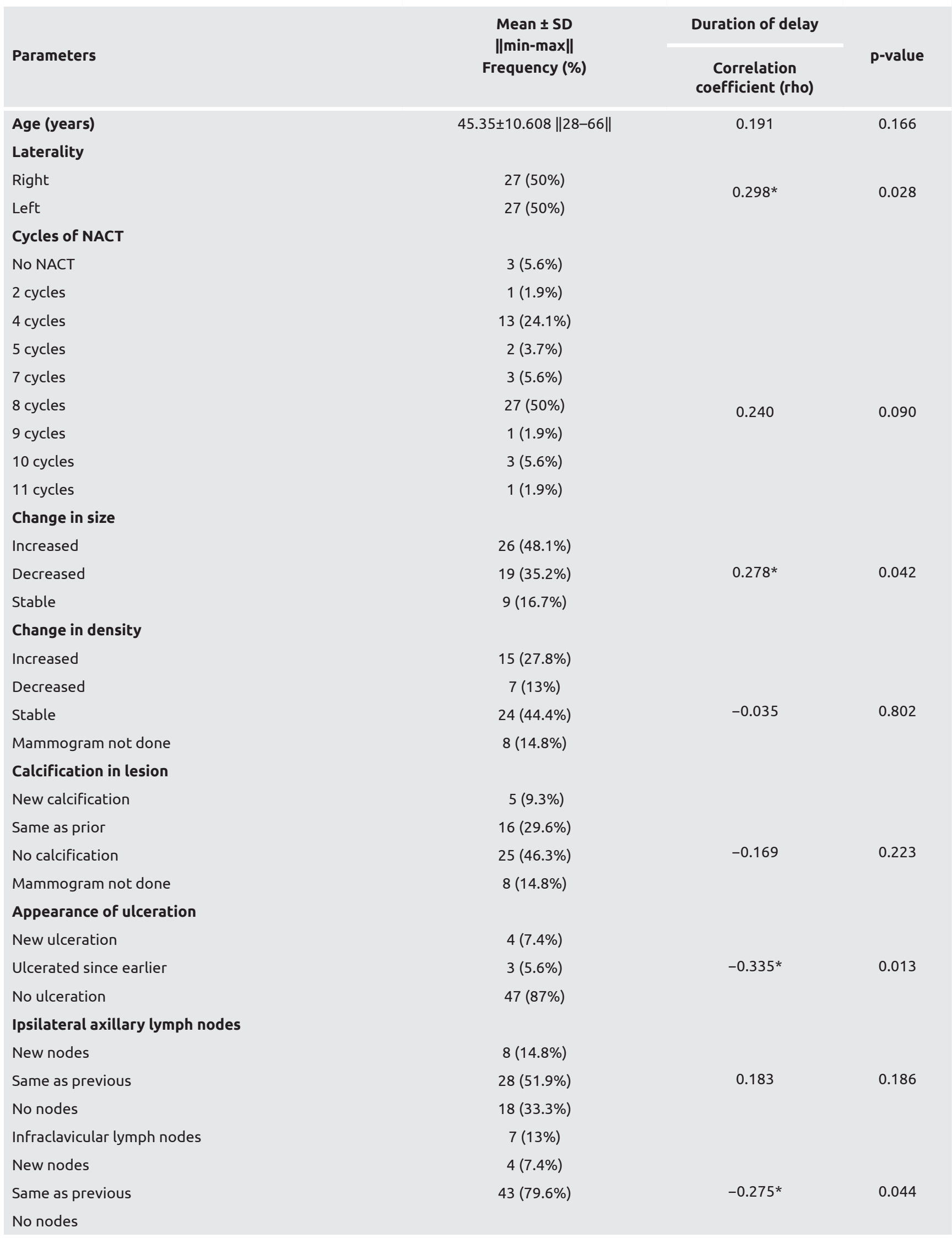


Table 2. Continued

$\begin{array}{cc} & \text { Mean } \pm \text { SD } \\ \text { Parameters } & \|\min -\max \| \\ \text { Frequency (\%) }\end{array}$

Duration of delay

Correlation

coefficient (rho)

\section{Supraclavicular lymph nodes}

New nodes

$2(3.7 \%)$

Same as prior

$4(7.4 \%)$

$-0.225$

0.101

No nodes

$48(88.9 \%)$

Contralateral side

New infiltrated axillary lymph node

$2(3.7 \%)$

No change

$47(87 \%)$

New malignant breast lesion

$5(9.3 \%)$

$-0.101$

0.166

Histological subtype

Luminal type $A$

19 (35.5\%)

ER/PR/HER2neu+ve

$8(14.8 \%)$

HER 2neu+ve

$10(18.5 \%)$

TNBC

0.219

0.111

Malignant phyllodes tumor

$2(3.7 \%)$

RECIST

Stable

Progressive disease

Partial response

$0.274^{*}$

0.045

p-value

Complete response

NACT: Neoadjuvant chemotherapy; COVID-19: Coronavirus disease-2019; ER: Estrogen receptor; PR: Progesterone receptor; HER2: Human epidermal growth factor receptor 2; SD: Standard deviation; min: Minimum; max: Maximum; RECIST: Response evaluation criteria in solid tumors

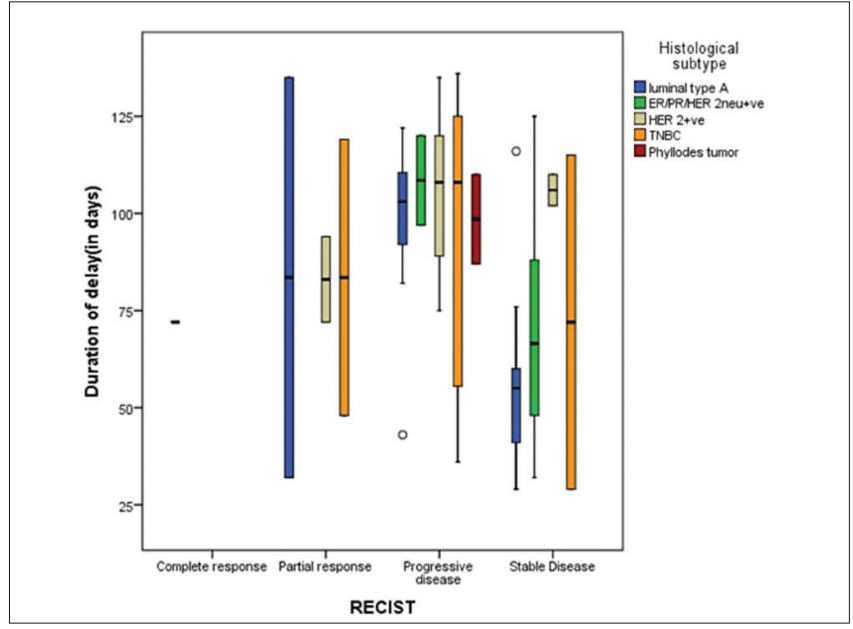

Figure 1. Clustered boxplot showing tumor response in relation to various histological subtypes of malignant breast lesions in postCOVID-19 period. We can see that complete response was seen only in $1(1.9 \%)$ case with luminal type A as histological subtype with delay in duration of 72 days. As the duration of delay increased, most of histological subtypes showed either progressive $(n=28,51.9 \%)$ or stable disease $(n=19,35.2 \%)$

COVID-19: Coronavirus disease-2019; LN: Lymph nodes; ER: Estrogen receptor; PR: Progesterone receptor; HER2: human epidermal growth factor receptor 2; TNBC: Triple-negative breast cancer; $n$ : Number; RECIST: Response evaluation criteria in solid tumors such as chemotherapy, radiation, and endocrine therapy, were used to pre-treat tumor (15). Due to this technique, mortality associated with breast cancer has reduced these days. Using neoadjuvant therapies, even all those cancers that are initially inoperable can be downgraded to fulfill operability criteria (8).

In breast cancer management, neoadjuvant therapies are being followed at almost all centers these days. Generally, a 3-month course is offered, and then the tumor is evaluated for reduction in volume (15). In our center, the patient is reevaluated with mammography and ultrasonography after completion of four and sometimes eight cycles of chemotherapy, and response evaluation is conducted. Prior to surgery, another follow-up imaging is performed.

According to the literature, the duration between the last neoadjuvant chemotherapy and surgery should not be more than 28 days. This gap of 28 days is necessary for overcoming the neutropenic window $(8,10)$. However, there are no clear-cut guidelines for the maximum duration allowed between the last cycle of neoadjuvant therapy and surgery, and different studies show different results (16-20). In our center, patients are generally operated post 2-3 weeks of the last chemotherapy cycle. However, due to the coronavirus pandemic, this time interval has increased. Most of the patients were able to visit the hospital after a median duration delay of 89 days (mode $=72$ days, mean $=85.76$ days). Thus, a repeat imaging with mammography and 
ultrasonography of all the patients who visited post-lockdown was performed to know the status of disease.

There are fewer studies which state that delayed duration of $>8$ weeks in neoadjuvant therapy and surgery is associated with poor outcomes (21). However, the reason of delay in their study was unknown.

Studies by Jazieh et al. (4) and Li et al. (6) have been carried out postcoronavirus pandemic, which clearly shows the inadvertent effects of delay in treatment of cancer patients. Every aspect of breast cancer management has been affected from screening to diagnosing and treatment $(2,6)$. Many studies have shown the effects of change of care of cancer patients on imaging modalities in the form of reduction in case volumes of mammography, ultrasonography, and breast MRI
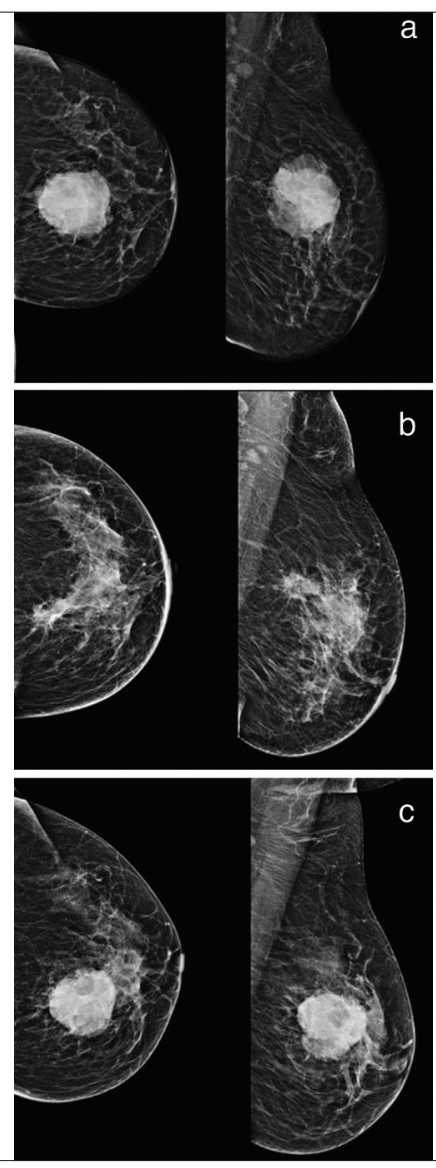

Figure 2. Mammogram images of a 52-year-old female patient who initially presented with lump in her left breast. HPE from the lesion was s/o invasive carcinoma grade III, ER/PR negative, and HER2 neu equivocal. (a) CC and MLO images done at baseline was s/o ovalshaped high-density lesion with microlobulated margins in the left breast in the upper inner quadrant. Few foci of microcalcification can also be seen within the lesion on CC view. Few equal-density lymph nodes with loss of hila can also be seen in the left axilla. (b) CC and MLO images done post 4 cycles of NACT during the pre-COVID-19 time at a duration delay of 10 days show reduction in lesion size along with mild reduction in density s/o partial response (RECIST 1.1). (c) CC and MLO images done after COVID-19 at a duration delay of approximately 115 days (3.8 months) showed s/o increase in size of the left breast lesion along with an increase in its density. The overall response assessment was s/o progressive disease (RECIST 1.1)

HPE: Histopathological Examination; HER2: Human epidermal growth factor receptor 2; CC: Craniocaudal; MLO: Mediolateral oblique; NACT: Neoadjuvant chemotherapy; COVID-19: Coronavirus disease-2019; ER: Estrogen receptor; PR:
$(2,7)$. However, so far, no study has shown what changes are actually observed on imaging in follow -up patients in the post-pandemic period. Moreover, none of the studies has shown changes in mammography density, appearance of new calcification, ulceration and lymph nodes, and their association with the histological subtype. This novel study aims to review these radiological changes observed on mammogram.

It can be clearly seen from our study that duration of delay in neoadjuvant therapy and surgery is associated with progression of diseases, worsening the outcome.

The main limitation of our study is short duration of the study. A small sample size could be collected in this short duration of study. Other shortcoming is that it is a single-centered study. A larger study with more samples may show more reliable results. Although patients who presented for the first time post-lockdown were not included in this study, they also presented with advanced disease post-lockdown as they were reluctant to visit hospitals during the lockdown. The reasons of delayed presentation also need to be studied so that they can be remedied in future. This may form a basis for further research.

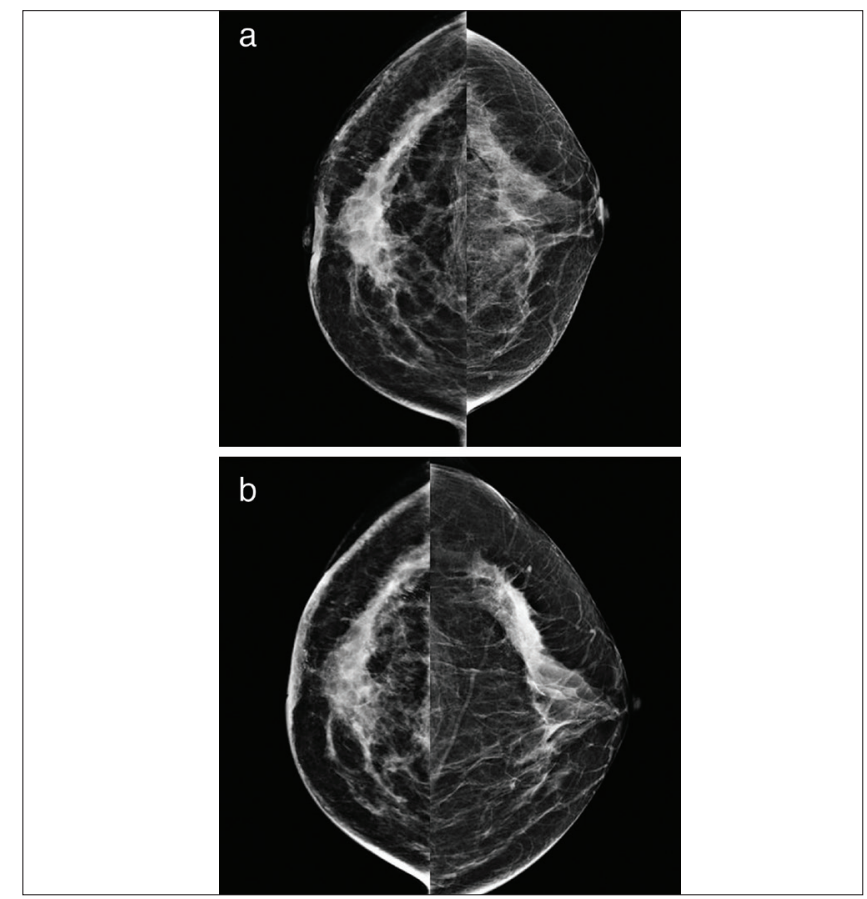

Figure 3. Mammogram images of a 50-year-old female patient who initially presented with lump in her right breast. HPE from the lesion was s/o invasive carcinoma grade III, ER positive, and PR/HER2neu negative. (a) CC image of right breast done at baseline was s/o an irregular iso- to high-density lesion showing spiculated margins in upper outer quadrant and retroareolar region, reaching up to the nipple, showing few fine pleomorphic microcalcifications within and in vicinity of the lesion with surrounding architectural distortion. The left breast was normal. (b) CC images done post 8 cycles of NACT during the post-COVID-19 time at a duration delay of 52 days show no significant change in the size of the right breast BIRADS 6 lesion; however there is appearance of new irregular-shaped high-density lesion with indistinct margins in the left breast upper outer quadrant. HPE from this lesion was malignant etiology. The overall response assessment was s/o progressive disease (RECIST 1.1)

HPE: Histopathological Examination; HER2: human epidermal growth factor receptor 2; CC: Craniocaudal; MLO: Mediolateral oblique; NACT: Neoadjuvant chemotherapy; COVID-19: Coronavirus disease-2019; ER: Estrogen receptor; PR: Progesterone receptor; BIRADS: Breast Imaging Reporting and Database System score; RECIST: Response evaluation criteria in solid tumors 
In conclusion, the coronavirus pandemic has severe impact on breast cancer management. A significant correlation was found in progression of disease with increase in duration of delay as seen during the pre- and post-COVID-19 time. A delay in neoadjuvant chemotherapy cycles and duration between the last cycle of neoadjuvant chemotherapy and surgery cause progression in cancer. This can be observed in mammogram and ultrasound. Delayed duration in surgery is clearly associated with increased size of lesion, increased mammography density, and appearance of ulceration in lesion. The appearance of new calcification, contralateral malignant breast lesion, and axillary and supra/infraclavicular lymph nodes were also seen. Furthermore, COVID-19 pandemic has had a devastating impact on breast cancer patients.

Ethics Committee Approval: Since this study is a retrospective study, ethics committee approval is not necessary.

Informed Consent: Informed consent was taken from the patient.

Peer-review: Externally peer-reviewed.

\section{Authorship Contributions}

Conception: A.S., B.R., N.C., S.R., P.S.; Design: G.K., A.K.; Supervision: A.K., P.S., S.C.; Materials: G.K., M.C.; Data Collection or Processing: G.K., A.D., S.C., P.G.; Analysis or Interpretation: G.K., A.K., S.C.; Literature Search: G.K., M.C., A.D., P.G.; Writing: G.K., M.C., A.K., B.R., N.C., S.R., P.S.

Conflict of Interest: No conflict of interest was declared by the authors.

Financial Disclosure: The authors declared that this study received no financial support.

\section{References}

1. COVID-19 pandemic lockdown in India. In: Wikipedia [Internet]. 2020. Available from: https://en.wikipedia.org/wiki/COVID-19_lockdown_ in_India [CrossRef]

2. Freer PE. The Impact of the COVID-19 pandemic on breast imaging. Radiol Clin North Am 2021; 59: 1-11. (PMID: 33222992) [CrossRef]

3. Broom A, Kenny K, Page A, Cort N, Lipp ES, Tan AC, et al. The paradoxical effects of COVID-19 on cancer care: current context and potential lasting impacts. Clin Cancer Res 2020; 26: 5809-5813. (PMID: 32816894) [CrossRef]

4. Jazieh AR, Akbulut H, Curigliano G, Rogado A, Alsharm AA, Razis $\mathrm{ED}$, et al. Impact of the COVID-19 pandemic on cancer care: a global collaborative Study. JCO Glob Oncol 2020; 6: 1428-1438. (PMID: 32986516) [CrossRef]

5. Veronesi P, Corso G. Impact of COVID-19 pandemic on clinical and surgical breast cancer management. EClinicalMedicine 2020; 26: 100523. (PMID: 32984788) [CrossRef]

6. Li J, Wang H, Geng C, Liu Z, Lin Y, Nie J, et al. Suboptimal declines and delays in early breast cancer treatment after COVID-19 quarantine restrictions in China: A national survey of 8397 patients in the first quarter of 2020. EClinicalMedicine 2020; 26: 100503. [CrossRef]

7. Sendur HN, Gultekin S, Cerit MN, Cindil E, Aslan AA, Karakoc E. Impacts of COVID-19 pandemic on breast imaging examinations. EJMI 2020; 4: 529-533. [CrossRef]
8. Bartsch R, Bergen E, Galid A. Current concepts and future directions in neoadjuvant chemotherapy of breast cancer. Memo 2018; 11: 199-203. (PMID: 30220926) [CrossRef]

9. Kaufmann M, Minckwitz G von, Bear HD, Buzdar A, McGale P, Bonnefoi $\mathrm{H}$, et al. Recommendations from an international expert panel on the use of neoadjuvant (primary) systemic treatment of operable breast cancer: new perspectives 2006. Ann Oncol 2007; 18: 1927-1934. (PMID: 17998286) [CrossRef]

10. Curigliano G, Burstein HJ, Winer EP, Gnant M, Dubsky P, Loibl S, et al. De-escalating and escalating treatments for early-stage breast cancer: the St. Gallen International Expert Consensus Conference on the primary therapy of early breast cancer 2017. Ann Oncol 2017; 28: 1700-1712. (PMID: 28838210) [CrossRef]

11. Fowler AM, Mankoff DA, Joe BN. Imaging neoadjuvant therapy response in breast cancer. Radiology 2017; 285: 358-375. (PMID: 29045232) [CrossRef]

12. Ollivier L, Balu-Maestro C, Leclère J. Imaging in evaluation of response to neoadjuvant breast cancer treatment. Cancer Imaging 2005; 5: 27-31. (PMID: 16154816) [CrossRef]

13. Eisenhauera EA, Therasseb P, Bogaertsc J, Schwartzd LH, Sargente D, Fordf R, et al. New response evaluation criteria in solid tumours: revised RECIST guideline (version 1.1) Eur J Cancer 2009; 45: 228-247. (PMID: 1909777) [CrossRef]

14. Müller C, Juhasz-Böss I, Schmidt G, Jungmann P, Solomayer EF, Breitbach GP, et al. Factors influencing the time to surgery after neoadjuvant chemotherapy in breast cancer patients. Arch Gynecol Obstet 2020; 301: 1055-1059. (PMID: 32170410) [CrossRef]

15. Selli C, Sims AH. Neoadjuvant therapy for breast cancer as a model for translational research. Breast Cancer (Auckl) 2019; 13: 1178223419829072. (PMID: 30814840) [CrossRef]

16. Yoo TK, Moon HG, Han W, Noh DY. Time interval of neoadjuvant chemotherapy to surgery in breast cancer: how long is acceptable? Gland Surg 2017; 6: 1-3 (PMID: 28210546) [CrossRef]

17. Chavez-MacGregor M, Clarke CA, Lichtensztajn DY, Giordano SH. Delayed initiation of adjuvant chemotherapy among patients with breast cancer. JAMA Oncol 2016; 2: 322-329. (PMID: 26659132) [CrossRef]

18. Yoo TK, Han W, Moon HG, Kim J, Lee JW, Kim MK, et al. Delay of treatment initiation does not adversely affect survival outcome in breast cancer, delay of treatment initiation does not adversely affect survival outcome in breast cancer. Cancer Res Treat 2015; 48: 962-969. (PMID: 26511801) [CrossRef]

19. Bleicher RJ, Ruth K, Sigurdson ER, Beck JR, Ross E, Wong YN, et al. Time to surgery and breast cancer survival in the United States. JAMA Oncol 2016; 2: 330-339. (PMID: 26659430) [CrossRef]

20. Lohrisch C, Paltiel C, Gelmon K, Speers C, Taylor S, Barnett J, et al. Impact on survival of time from definitive surgery to initiation of adjuvant chemotherapy for early-stage breast Cancer. J Clin Oncol 2006; 24: 4888-4894. (PMID: 17015884) [CrossRef]

21. Sanford RA, Lei X, Barcenas CH, Mittendorf EA, Caudle AS, Valero V, et al. Impact of time from completion of neoadjuvant chemotherapy to surgery on survival outcomes in breast cancer patients. Ann Surg Oncol 2016; 23: 1515-1521. (PMID: 26678405) [CrossRef] 